\title{
Primary progressive apraxia: a syndrome difficult to categorize
}

\author{
Apraxia progressiva primária: uma condição difícil de classificar \\ Ricardo Krause Martinez de Souza', Samanta Fabricio Blattes da Rocha², Marcela Ferreira Cordellini \\ Ronaldo Pereira Vosgerau4, Pedro André Kowacs²
}

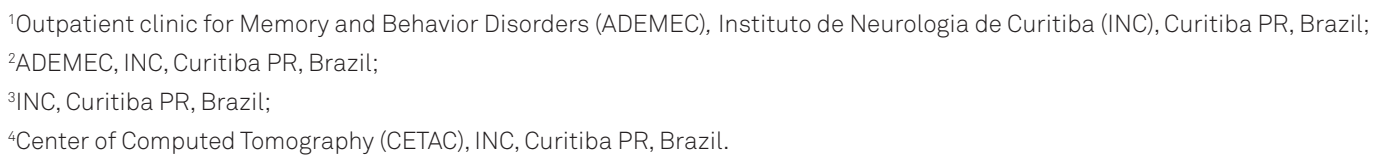

Diagnoses have nosological, therapeutic and prognostic goals. Among the dementias, primary progressive apraxia has an uncertain etiology, presenting as a variant of Pick's disease $^{1}$ or other nosological entities ${ }^{2}$. The syndrome usually manifests in the sixth decade of life, and its clinical diagnosis depends on the types of apraxia manifested initially. This letter was motivated by the following case:

\section{CASE}

A right-handed, illiterate, 70-year-old female farmhand presented in 2007 with instability, anterior trunk flexion, short steps and frequent falls, which evolved to apraxic gait. In 2008 she began to need assistance for walking and activities such as getting dressed, combing her hair or brushing her teeth. In 2009, she started to use a wheelchair because of her apraxic gait. She did not present with memory deficit or behavioral change, but had urinary incontinence. Physical examination revealed interosseous muscle atrophy and contractures in both hands, palmomental reflex, and appendicular hypertonia with spasticity that was more prominent on the left side. She presented with dressing, ideational and ideomotor apraxia without impairment of orientation, memory, speech, gnosis or executive functions. Laboratory tests were unremarkable. Brain MRI revealed loss of cortical/subcortical differentiation in the superior parietal lobes and supplementary motor area that was more pronounced on the left side (Figure). There was no response to baclofen, levodopa/ benserazide or amantadine. After three years of evolution of the disease, with progressive worsening of apraxic features, the patient was followed-up for approximately one year after initial evaluation before being lost. During this period her cognitive functions remained stable, but a progressive

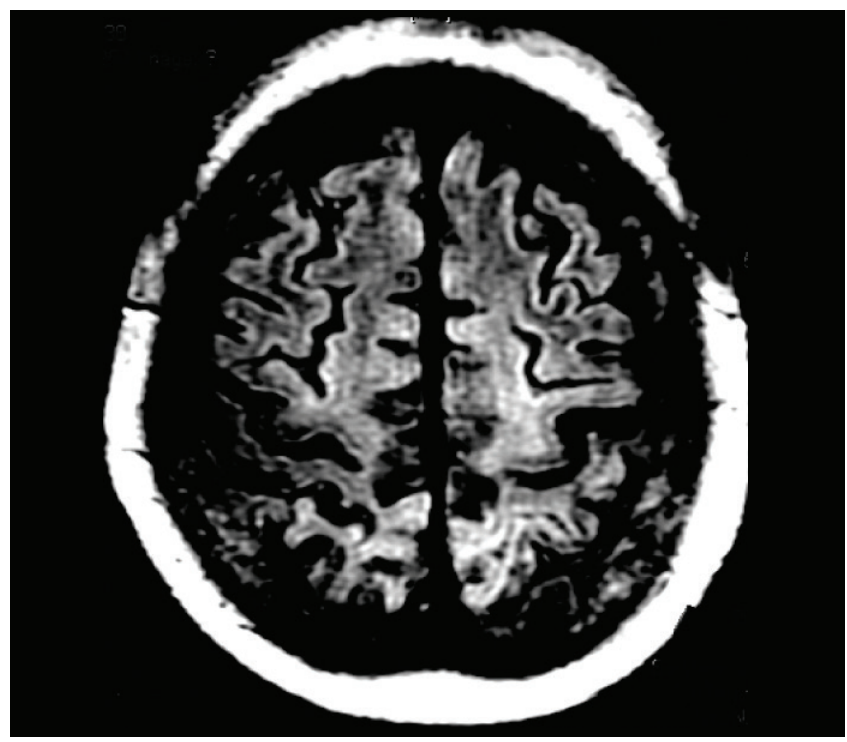

Figure. Brain MRI showing loss of cortical/subcortical differentiation in the superior parietal lobes and supplementary motor area that was more pronounced on the left side.

worsening of the various apraxic dysfunctions was observed. Unfortunately, we were not able to extend the investigation by measuring biomarkers, for instance, due to the lack of financial support. However, our hypothesis of a tau protein-related neuropathology should be taken into consideration in the light of previous evidence ${ }^{1-4}$.

\section{DISCUSSION}

Primary progressive apraxia is a rare condition, frequently accompanied by extrapyramidal signs and dyscalculia ${ }^{2}$. Fukui et al. published a case with pathological findings compatible with Pick's disease ${ }^{1}$. Our patient presented with multiple 
apraxia, spasticity and primitive reflexes. In the absence of cognitive and memory impairment, a diagnosis of Alzheimer's disease is unlikely ${ }^{3}$, as well as the diagnosis of corticobasal degeneration, for which extrapyramidal signs are required ${ }^{4}$.

It is possible that primary progressive apraxia is a tau protein-related neuropathology whose etiology cannot always be categorized based on clinical findings, as these may be compatible with Pick's disease, Alzheimer's disease or corticobasal degeneration. Our patient presented with clinical signs and symptoms incompatible with these conditions, suggesting that more specific and atypical cases may exist.

\section{References}

1. Fukui T, Sugita K, Kawamura M, Shiota J, Nakano I.Primary progressive apraxia in Pick's disease: a clinicopathologic study. Neurology 1996;47:467-473.

2. Kawamura M, Mochizuki S. Primary progressive apraxia. Neuropathology 1999; 19:249-258.
3. McKhann GM, Knopman DS, Chertkow H, et al. The diagnosis of dementia due to Alzheimer's disease: recommendations from the National Institute on Aging-Alzheimer's Association workgroups on diagnostic guidelines for Alzheimer's disease. Alzheimers Dement 2011;7:263-269.

4. Lang AE, Riley DE BC. Corticobasal ganglionic degeneration. In: Calne DB (Ed). Philadelphia, W.B. Saunders Company; 1994:877-894. 